\title{
Flaxseed oil, a fish oil challenger?
}

Jean-Marc MAURETTE

53 rue Auguste Buisson

92250 La Garenne-Colombes

France

<jm.maurette@orange.fr>

\begin{abstract}
The too low level of omega-3 intake in the modern diet is a fact which is worrying health professional and authorities. Among the "traditional" recommendation for a safe and equilibrated diet, recommending food containing omega-3, is there, out of the inescapable fish oil leading to some digestive discomforts, any alternative? Two human clinical trials conducted to verify the effective efficiency of this re equilibrated diet on various skin parameters (hydration, surface evaluation, inflammation) are reviewed here. The results of the clinical trials on various skin parameters (hydration, trans epidermal water loss, roughness, scaling, superficial inflammation and blood flow) were quite convincing about the interest of such a supplementation.

Key words: PUFA, skin, hydration, inflammation, blood flow
\end{abstract}

Among this wide range of activities, omega-3 has a great impact on skin physiology. Basic skin functions such as the barrier function, temperature regulation, photo protection, water homeostasis are impacted by supplementary or dietary intervention $[12,13]$. The modulation of these functions is generally coupled with changes in skin structure which produce some changes in skin appearance.

Linoleic acid (AA), $\gamma$-linolenic acid (GLA) or $\alpha$-linolenic acid (ALA) play a role in cellular signaling interfering with eicosanoids pathways or influencing the regulation of gene expression $[13,14]$.

Further, epidermal lipids are known to play a crucial role in the mediation of desquamation and the deficiency of essential fatty acids seems to be involved in cutaneous scaling disorders (xerosis, psoriasis, atopic dermatitis) [15].

Two recent orientation studies were performed in dermatology, focusing on skin hydration and skin sensitivity. They were successively designed and performed in order to verify under real conditions of use whether or not the oral administration of ALA (through flaxseed oil, 55\% ALA) have any influence on these skin conditions.

In other words, among the general properties of PUFAs, is it possible to determine some concrete facts showing the interest of consuming reasonable amounts of omega- 3 and to see an improvement of skin health conditions. This is the aim of the two study performed which conditions and results will be described in this paper $[16,17]$.

Physiological activity of flaxseed oil: two clinical trials in dermatology.

\section{Skin sensitivity I}

\section{Study design}

This first study was carried out as a monocentric, double-blind, randomized and placebo controlled application test on 26 healthy volunteers with dry (Corneometer value $<40 \mathrm{au}^{1}$ [18]) and sensitive skin. Subjects were divided into two subgroups of 13 (1 verum - 1 reference group).

Verum was composed of $555.32 \mathrm{mg}$ of flaxseed oil (i.e. $\geq 289 \mathrm{mg} \mathrm{ALA} /$ capsule) associated with stabilizing antioxidants (D- $\alpha$-tocopherol and Rosemary extract). The daily dose of ALA was of at least 1,155 mg. Reference was composed of $560 \mathrm{mg}$ safflower oil, with a minimum LA content of $70 \%$ (i.e. $\geq 392 \mathrm{mg} /$ capsule).

The study lasted for 12 weeks, dosage regimen being 4 capsules/day ( 2 at breakfast; 2 at diner). Parameters of the study were measured before supplementation and at weeks 6 and 12 (end point).

\section{Methods}

Skin parameters were evaluated by measurement of skin hydration (Corneometer CM825 ${ }^{\circledR}$ - Courage \& Khazaka), transepidermal water loss (TEWL, barrier function of the skin) (Tewameter TM $300^{\circledR}$ - Courage \& Khazaka) $[18,19]$ and skin surface structure (SELS, Visios$\mathrm{can}^{\circledR}$ - Courage \& Khazaka) for the evaluation of the following items: roughness, scaling, smoothness and wrinkles [20].

The sensitivity of the skin was assessed by the Nicotinate irritation test according to Primavera \& Berardesca [21]. Measurements were

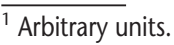




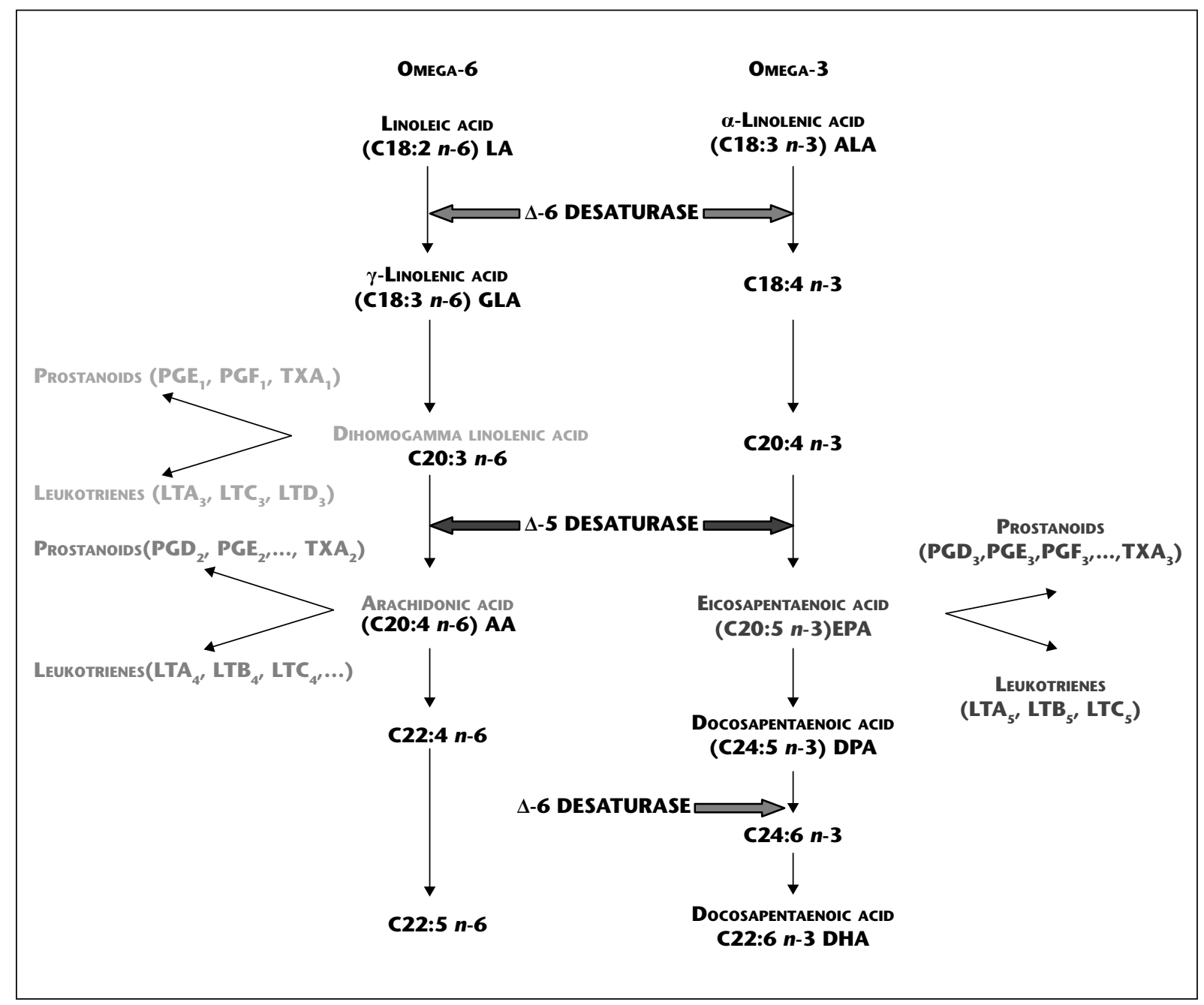

Figure 1. Biosynthesis of the $\omega-6$ and $\omega-3$ PUFA's series.

performed on the inner forearm of the volunteers. After application of nicotinate $(0.25 \%$, $5 \mu \mathrm{L} / \mathrm{cm}^{2}$ ), inducing a local inflammation, reddening of the skin was measured by chromametry as " $a$ " value (Minolta CR $300^{\circledR}$ ). Additionally, capillary surface blood flow was measured by Laser-Doppler-Flowmetry (O2C-System, Lea Instruments, Giessen, Germany).

\section{Statistics}

For all parameters and all time points (week 0 , week 6, week 12) descriptive statistics (mean, standard deviation, minimum, lower quartile, median, upper quartile and maximum) were calculated. For all parameters pre-post differences were calculated and analyzed descriptively. For the pre-post differences the two treatment groups were compared using the Wilcoxon rank-sum test

Within the two treatment groups each combination of two time points was compared using the Wilcoxon signed-rank test.

\section{Results}

In summary this orientation study clearly demonstrates the effect of the supplementation by ALA ( $1.15 \mathrm{~g} /$ day on the average) on skin parameters and inflammation induced by the nicotinate test as shown in table 1.

The following results were observed.

\section{Treatment A: Flaxseed oil}

After 6 weeks of treatment:

- For treatment A, statistically significant changes $(p<0.05)$ for the parameters "Blood Flow" (decrease) and "SELS: roughness" (increase) were observed, compared to baseline. - For treatment B a statistically significant increase $(p<0.05)$ for the parameter "SELS: roughness" was observed compared to week 0 .

After 12 weeks of treatment:

- For treatment $A$ a statistically significant increase $(p<0.05)$ for the parameters skin hydration and smoothness was observed after twelve weeks compared to week 0 .
A statistically significant decrease $(p<0.05)$ for the parameters TEWL, roughness, scaling, redness $a$-value and blood flow, was observed after twelve weeks compared to week 0 .

\section{Treatment B: Safflower oil}

After 6 weeks of treatment:

- Treatment $B$ showed a statistically significant increase $(p<0.05)$ for the parameter "skin hydration" (+13\%) compared to week 0 .

After 12 weeks of treatment:

- A statistically significant decrease $(p<0.05)$ for the parameter roughness was observed after twelve weeks compared to week 0 .

\section{Comparison within groups ( $A$ vs $B$ )}

For the pre-post differences "week 0 - week 6" the comparison of the two treatment groups showed statistically significant differences ( $p$ $<0.05$ ) in favor of treatment A for the parameter blood flow. 
Table 1. Percentual changes vs T0 after 6 and 12 weeks.

\begin{tabular}{|c|c|c|c|c|}
\hline & \multicolumn{2}{|c|}{ Flaxseed oil (Omega-3) - A } & \multicolumn{2}{|c|}{ Safflower oil (Omega-6) - B } \\
\hline & Week 6 & Week 12 & Week 6 & Week 12 \\
\hline Hydration & $+7 \%$ (ns) & $+39 \% \%^{a, b}$ & $-2 \%(n s)$ & $+13 \%{ }^{a}$ \\
\hline TEWL & $-21 \%{ }^{a}$ & $-31 \%{ }^{a}$ & $-6 \%(n s)$ & $-12 \%$ (ns) \\
\hline \multicolumn{5}{|l|}{ SELS } \\
\hline Roughness & $-22 \%{ }^{a}$ & $-30 \%{ }^{a, b}$ & $-10 \%{ }^{a}$ & $-10 \%{ }^{a}$ \\
\hline Scaling & $-15 \%$ (ns) & $-31 \%^{a}$ & $-6 \%$ (ns) & $-14 \%$ (ns) \\
\hline Smoothness & $+3 \%(\mathrm{~ns})$ & $+7 \%^{\mathrm{a}}$ & $-1 \%(n s)$ & $+2 \%(\mathrm{~ns})$ \\
\hline Wrinkles & $-0.4 \%$ (ns) & $+2 \%$ (ns) & $+2 \%$ (ns) & $+4 \%$ (ns) \\
\hline \multicolumn{5}{|l|}{ Nicotinate test } \\
\hline Redness (a-values) & $-22 \%$ (ns) & $-48 \%{ }^{a, b}$ & $-0.2 \%$ (ns) & $-7 \%$ (ns) \\
\hline Blood flow & $-34 \% \%^{a, b}$ & $-66 \%{ }^{a, b}$ & $+11 \%$ (ns) & $+7 \%(\mathrm{~ns})$ \\
\hline
\end{tabular}

ns : not significant.

a Significant ( $p<0.05$, Wilcoxon Rank sum Test).

${ }^{b}$ A significantly different from $B(p<0.05$, Wilcoxon signed rank test).
For the pre-post differences "week 0 - week $12^{\prime \prime}$ the comparison of the two treatment groups showed statistically significant differences $(p<0.05)$ in favor of treatment $A$ for the parameters skin hydration, roughness, redness a-value, blood flow.

For all the other parameters, on the pre-post differences, no statistically significant differences can be shown.

\section{Skin sensitivity II}

In the second study the effects of flaxseed oil were compared with the one of a reference product, the borage oil, known to have hydrating properties on the skin [22].

It seemed interesting to have an idea of the performances of both oils on the previously studied parameters as described in the Sensitive Skin I study.

\section{Study design}

The study was carried out as a monocentric, randomized, double-blind, placebo-controlled application test in three parallel groups of fifteen subjects each (45 healthy non-smoking women, between 18 and 65 years) with dry (Corneometer value < 40 au [18] and sensitive skin.

Exclusion criteria were: pregnancy and breastfeeding, history of fat malabsorption, liver diseases, and diseases regarding lipid metabolism or any photosensitizing disorder. BMl of the participants were between 18 to $25 \mathrm{~kg} / \mathrm{m}^{2}$. They did not take lipid or vitamin supplements or any other medication.

Daily doses amounted to $2.22 \mathrm{~g}$ flaxseed (1,100 mg ALA C18:3n-3) or borage oil (480 mg GLA C18:3 n-6); in both groups, intake of D- $\alpha$-tocopherol was $10 \mathrm{mg} / \mathrm{d}$.

Placebo was made of $565 \mathrm{mg}$ medium chain triglycerides (caprilic and capric acid). Daily intake of the placebo was of $2.26 \mathrm{~g}$.
The study lasted for 12 weeks, dosage regimen being 4 capsules/day: 2 at breakfast; 2 at diner.

\section{Methods}

Parameters of the study were measured before supplementation and at weeks 6 and 12 (end point): Skin hydration in arbitrary units (Corneometer ${\mathrm{C} 285^{\circledR}}^{\circledR}$ ), TEWL in $\mathrm{g} / \mathrm{h}$ per $\mathrm{m}^{2}$ (TEWAMeter TM 300 - Courage \& Khazaka Electronics $[18,19]$, SELS (Visioscan - Courage \& Khazaka) [20] for the evaluation of the following items: roughness, scaling, smoothness and wrinkles. Nicotinate irritation was used to test the sensitivity of the skin according to Primavera et al. [21]. Measurements were performed on the inner forearm of the volunteers. No treatment with any ointment on the tested areas was allowed during the whole study. Nicotinate $\left(0.25 \% ; 5 \mu \mathrm{L} / \mathrm{cm}^{2}\right)$ was applied inducing an inflammation of the skin; reddening was measured by chromametry (Minolta CR 300, Ahrensburg, Germany) as "a-value" before and after treatment. Erythema intensity is given as $\Delta$ a-value; a-value after treatment minus a-value before treatment. Additionally, capillary blood flow was determined by Laser-DopplerFlowmetry (O2C-System, Lea Instruments, Giessen, Germany) in the irritated area.

\section{Statistics}

For all parameters and all time points (week 0 , week 6, week 12) descriptive statistics (mean, standard deviation, minimum, lower quartile, median, upper quartile and maximum) were calculated. For all parameters pre-post differences were calculated and analyzed descriptively.

For the pre-post differences the two treatment groups were compared using the Wilcoxon rank-sum test. Within the two treatment groups each combination of two time points was compared using the Wilcoxon signed-rank test. In addition, the two pre-post differences Week 6/Week 0 and Week 12/Week 0 were compared by an ANOVA with parameter treatment as the independent variable. The least square means for the pre-post differences and the respective $95 \%$ confidence intervals were calculated. These confidence intervals were used to assess whether the pre-post difference was significantly different from [17].

\section{Results}

The effects of the supplementation by ALA or GLA on skin parameters and inflammation induced by the nicotinate test are shown in table 2.

One can see that the results of this second study are consistent with the ones of the Skin
Table 2. Percentual changes vs T0 after 6 and 12 weeks.

\begin{tabular}{|c|c|c|c|c|c|c|}
\hline & \multicolumn{2}{|c|}{$\begin{array}{c}\text { Flaxseed oil } \\
\text { (Omega-3) - A }\end{array}$} & \multicolumn{2}{|c|}{$\begin{array}{c}\text { Borage oil } \\
\text { (Omega-6) - B }\end{array}$} & \multicolumn{2}{|c|}{ Placebo - C } \\
\hline & Week 6 & Week 12 & Week 6 & Week 12 & Week 6 & Week 12 \\
\hline Hydration & $+12 \%^{\mathrm{a}}$ & $+19 \%^{\mathrm{a}}$ & $+7.9 \%$ (ns) & $+16.6 \%{ }^{a}$ & $+3 \%(n s)$ & $+9 \% \%^{a}$ \\
\hline TEWL & $-8 \%^{a}$ & $-26 \%{ }^{a}$ & $-9 \%{ }^{a, d}$ & $-11.2 \%{ }^{a, d}$ & $-0.6 \%$ (ns) & $-1.2 \%$ (ns) \\
\hline \multicolumn{7}{|l|}{ SELS } \\
\hline Roughness & $-15 \%^{a}$ & $-34 \% \%^{a, b, c}$ & $-7.6 \%$ (ns) & $-14.7 \%{ }^{a}$ & $-0.61 \%$ (ns) & $+1 \%$ (ns) \\
\hline Scaling & $-29 \% \%^{a, b}$ & $-35 \%{ }^{a, b}$ & $-25.1 \%$ (ns) & $-26.5 \%^{a}$ & $-4.3 \%$ (ns) & $-12.6 \%$ (ns) \\
\hline Smoothness & $+5 \%$ (ns) & $+4 \%^{\mathrm{a}}$ & $-0.2 \%$ (ns) & $+2.6 \%$ (ns) & $-4.3 \% \%^{a}$ & $-2.3 \%$ (ns) \\
\hline Wrinkles & $-1 \%$ (ns) & $0 \%$ (ns) & $+0.8 \%$ (ns) & $+1.1 \%$ (ns) & $+1.5 \%$ (ns) & $+1.7 \%$ (ns) \\
\hline \multicolumn{7}{|l|}{ Nicotinate test } \\
\hline Redness (a-values) & $-32 \%{ }^{a}$ & $-45 \%^{a}$ & $-29.9 \%{ }^{a}$ & $-35.1 \%{ }^{a, d}$ & $0 \%$ (ns) & $-2 \%(n s)$ \\
\hline Blood flow & $-34 \%^{a}$ & $-\mathbf{8 1} \%^{a, b, c}$ & $-28 \%^{a}$ & $-33.8 \%^{a}$ & $-10 \%$ (ns) & $-8.5 \%$ (ns) \\
\hline
\end{tabular}

ns: not significant.

a Significant ( $p<0.05$, Wilcoxon Rank sum Test).

${ }^{\mathrm{b}}$ A significantly different from $C(p<0.05$, Wilcoxon signed rank test).

${ }^{\mathrm{C}}$ A significantly different from $B(p<0.05$, Wilcoxon signed rank test).

${ }^{d} B$ significantly different from $C(p<0.05$, Wilcoxon signed rank test). 
Sensitivity I study despite the limited number of healthy volunteers in each study. The data are of the same order of magnitude for the parameters TEWL, SELS, redness and blood flow for both flaxseed oil groups. The results on hydration were in both cases significantly different from time 0 inside of each group. However, in this second study, hydration was not significantly different from the corresponding placebo group after 12 weeks of treatment $(+19 \%$ vs. $+9 \%)$.

It is also interesting to note that significant effects are seen already at week 6 (hydration, TEWL, roughness, scaling, redness and blood flow) which was less clear for study I were significant differences from time 0 at 6 weeks were reached for TEWL, roughness and blood flow only.

\section{Discussion}

Flaxseed oil, cold pressed from the seeds of the flax plant (Linum usitatissimum), is one of richest sources of $n-3$ fatty acids (FA) in the vegetal world. With more than $50 \%$ of total FA, ALA is predominant in the oil which also contains the n-6 Linoleic acid (16\%) and the monounsaturated oleic acid (20\%) as major constituents (table 3). The flaxseed oil supplement used in the present study is typical with respect to its composition of fatty acids [23]. Borage oil is pressed from the seeds of the borage plant (Borago officinalis) and rich in the $\mathrm{n}-6$ fatty acids GLA (22\%) and LA (39\%); oleic acid (15\%) is also present in quite high amounts (table 3). Its composition is also typical regarding its FA pattern [23].

Facing the lack of omega- 3 consumption in the modern diet as shown by $\mathrm{N}$. Combe et al (2001) [24], it was interesting to see if oils of vegetable origin may have a significant impact

Table 3. Fatty acid composition (\% of total fatty acids) (From De Spirt et al. 2008 [17]).

\begin{tabular}{|cccc|}
\hline Fatty acid & Flaxseed oil & Borage oil & Placebo \\
\hline $8: 0$ & 0.00 & 0.00 & 36.9 \\
$10: 0$ & 0.00 & 0.00 & 21.8 \\
$14: 0$ & 4.73 & 1.87 & 0.24 \\
$18: 0$ & 4.12 & 2.82 & 0.00 \\
$18: 1 n-9$ & 19.6 & 15.0 & 0.60 \\
$18: 2 n-6$ & 16.0 & 38.7 & 0.00 \\
$18: 3 n-6$ & 0.00 & 21.6 & 0.18 \\
$18: 3 n-3$ & 52.8 & 0.38 & 0.00 \\
$20: 0$ & 0.16 & 0.21 & 0.00 \\
$20: 1$ & 0.20 & 3.95 & 0.00 \\
$20: 2$ & 0.00 & 0.23 & 0.00 \\
$22: 1$ & 0.00 & 2.23 & 0.00 \\
$24: 0$ & 0.00 & 0.00 & 0.00 \\
$24: 1$ & 0.00 & 1.30 & 0.00 \\
Non-identified fatty acids & 2.39 & 11.7 & 38.3 \\
\hline
\end{tabular}

The use of flaxseed oil may also contribute to restore a better ratio Omega 3/Omega 6 and further studies would be necessary with greater populations and on a longer period to appreciate the full benefits suggested in these two orientation studies.

However, it should be pointed out that flaxseed oil, despite its interest as shown in this paper, cannot be a substitute to a healthy and equilibrated diet.

Due to the lack of omega- 3 in the western food, to find another way to consume Omega 3 from natural origin may have sense, nutrition wise.

As shown by A. Morise et al. [25], absorption and storage of ALA is very efficient in the hamster. It powerfully increased EPA. However dietary ALA failed to increase DHA, but decreased AA. The authors concluded that dietary supply of ALA was a very efficient means of improving the balance omega-6/omega-3 (AA/EPA) in Red Blood Cells and cardiomyocytes.

It has also been shown (unpublished data) a significant bioconversion of ALA into EPA in Sensitive Skin I for the flaxseed oil group at week 12 , compared to the reference group (safflower oil).

The two studies presented in this article confirm this concept in showing interesting results in the field of skin hydration, inflammation and skin surface structure with acceptable doses of flaxseed oil compatible with the recommended daily allowances published by the French Food Safety Agency (AFSSA [26]) amounting at $1.6 \mathrm{~g} /$ day for non pregnant adult females.

Furthermore, the consumption of flaxseed oil has been recognized as an acceptable dietary practice by AFSSA in 2006 as far as flaxseed oil is consumed exclusively cold and with less than $1 \%$ of all trans fatty acids [27]. 
12. AFAQ F, MUKHTAR H. Botanical antioxidants in the prevention of photocarcinogenesis and photoaging. Exp Dermatol 2006; 15: 678-84.

13. SIES H, STAHLW. Nutritional protection against skin damage from sunlight. Annu Rev Nutr 2004; 24: 173-200.

14. ZIBOH VA, MILLER CC, CHO Y. Metabolism of polyunsaturated fatty acids by skin epidermal enzymes: generation of antiinflammatory and antiproliferative metabolites. Am / Clin Nutr 2000; 71: 361S-366S.

15. PROKSCHE, HOLLERAN W, MENON GK, ELIAS PM, FEINGOLD KR. Barrier function regulates epidermal lipid and DNA synthesis. $\mathrm{Br}$ I Dermatol 1993; 128: 473-82.

16. HEINRICH U, WIEBUSCH M, TRONNIER H, SIES H, STAHL W, BÉJOT M, MAURETTE JM. Supplementation of linoleic acid improves skin barrier function and skin physiological parameters. 16th EADV Congress, Vienna, P312. 2007.
17. DE SPIRT S, STAHL W, TRONNIER H, ET AL. Intervention with flaxseed and borage oil supplements modulates skin condition in women. BJN 2008; On line version p. 1-6.

18. HEINRICH U, KOOP U, LENEVEU-DUCHEMIN MC, ET AL. Multicenter comparison of skin hydration in terms of physical-, physiological- and product dependent parameters by the capacitive method (Corneometer CM 825). Intern / Cosm Sci 2003; 25: 45-53.

19. RODRIGUES LM, PINTO PC, MAGRO JM, FERNANDES M, ALVES J. Exploring the influence of skin perfusion on transepidermal water loss. Skin Res and Techn 2004; 10: 257-62.

20. TRONNIER H, WIEBUSCH M, HEINRICH U STUTE R. Surface evaluation of living skin. $A d v$ Exp Med Biol 1999; 455: 507-16.

21. PRIMAVERA G, BERARDESCA E. Sensitive skin: Mechanism and diagnosis. Int / Cosmet Sci 2005; 27: 1-10.
22. BROSCHE T, PLATT D. Effect of borage oil consumption on fatty acid metabolism, transepidermal waterloss and skin parameters in elderly people. Arch Gerontol Geriatr 2000; 30(2): 139. 50.

23. SANDERS TA, YOUNGER KM. The effect of dietary supplements of Omega 3 polyunsaturated fatty acids on the fatty acid composition of platelets and plasma choline phosphoglycerides. Br / Nutr 1981; 45: 613-6.

24. COMBE N, BOUE C. Apports alimentaires en acides linoléique et alpha-linolénique d'une population d'Aquitaine. OCL 2001; 8(2): 11821.

25. MORISE A, COMBE N, BOUE C, ET AL. Dose effect of alpha-Linolenic acid on PUFA conversion, bioavailability, and storage in the hamster. Lipids 2004; 39(4): 325-34.

26. AFSSA. Les apports nutritionnels conseillés pour la population française. Paris: Tec \& Doc, tab. 1, p. 67. 2007.

27. AVIS AFSSA 2004-SA-0213. 25 juillet 2006. 